\title{
Investigation of External Charges Effects on Piezoelectric ZnO Nanogenerator
}

\author{
Samira Fathi, Tahereh Fanaei Sheikholeslami* \\ Electronic Department, Faculty of Electrical and Computer Engineering, University of Sistan and Baluchestan, \\ Zahedan, Iran
}

(Received 15 March 2016; revised manuscript received 12 June 2016; published online 21 June 2016)

\begin{abstract}
Piezopotential generation in semiconductive $\mathrm{ZnO}$ nanowire (NW), oriented along the c-axis [0001], is significantly affected by free charge carriers within the ZnO NW. In this paper, the effect of free carriers' distribution in semiconductive $\mathrm{ZnO}$ nanowire is investigated, using a Finite Element Method (FEM). The mentioned effect leads to modification of the conduction band variation, carrier concentration profiles, and eventually, the magnitude and distribution of the piezoelectric potential. The impact of free charge carriers shows that the negative potential distributed at the tip of $\mathrm{ZnO} \mathrm{NW}$ is decreased from $V=-270 \mathrm{mv}$ for the donor concentration $N D=1 \times 10^{15} \mathrm{C} / \mathrm{m}^{3}$ to the $V=-25 \mathrm{mV}$, in presence of the donor concentration of $N D=1 \times 10^{18} \mathrm{C} / \mathrm{m}^{3}$. With selecting the appropriate electrical boundary conditions and applying the surface charges density at the top of the nanowire, the potential reduction is compensated. The electrostatic effect leads to a significant enhancement of the piezoelectric potential. The results are well shown the interplay of volume and surface charges and their influence on performance of nanogenerator, and so are crucial for designing of nanogenerators with high piezoelectric potential and good efficiency.
\end{abstract}

Keywords: Free charge carrier, Mechanical energy harvesting, Piezoelectric nanogenerator, Piezoelectric potential, Surface charge density, $\mathrm{ZnO}$ nanowire.

DOI: 10.21272 /jnep.8(2).02047

PACS numbers: 81.07.Gf, 85.50. - n

\section{INTRODUCTION}

Mechanical energy, as the most abundant and popular energies in our daily life, has attracted a lot of attention in energy harvesting technology. Mechanical energy harvesting, using existed nanotechnologies, offers a promising solution to build self-powered nanosystems with ultra small size, low-weight, independent and sustainable power supply with steady operation and long lifetime [1].

Piezoelectric zinc oxide ( $\mathrm{ZnO})$ nanogenerators (NGs) were first introduced by Wang and Song in 2006 [2]. Their nanogenerator, employed with vertically grown $\mathrm{ZnO}$ nanowires (NWs), converted the mechanical forces to the electrical voltage, when an AFM's tip swept across the nanowires. During the last decade, various inventions in piezoelectric materials, structures, substrates and also, in the synthesis techniques for the piezoelectric nanomaterial production have made huge progress in improving the performance of the piezoelectric NGs [3]. Various semiconductor piezoelectric nanostructured materials like $\mathrm{ZnO}$, GaN, PZT, BaTiO3, $\mathrm{NaNbO} 3$ and PVDF have been used as the building blocks for the piezoelectric NGs [4]. Among them, nanostructured $\mathrm{ZnO}$ is an attractive semiconductive piezoelectric material with many functional properties and three key advantages. First, $\mathrm{ZnO}$ exhibits both semiconducting and piezoelectric properties. Second, $\mathrm{ZnO}$ is nontoxic and biocompatible. Third, the methods for synthesize of $\mathrm{ZnO}$ are easy with low-cost processes [2]. Also $\mathrm{ZnO}$ has attracted much interest, in the last few years, because of its enhanced piezoelectric coefficient and low stiffness at nanometer scale [5].

So far, a lot of considerable efforts have been made to understand the behavior of $\mathrm{ZnO}$ nanowire, and to improve the performance and efficiency of the piezoe- lectric $\mathrm{ZnO}$ NGs. One of the most effective parameters on magnitude and distribution of the piezoelectric potential is the existence of free charge carriers inside the $\mathrm{ZnO}$ [6]. The $\mathrm{ZnO}$ is intrinsically a semiconductor material and the value of impurity plays a major role in conductivity of $\mathrm{ZnO}$. In principle, the free charges lead to partially screen the piezoelectric polarization charges. The latter, as an undesirable effect, reduces the magnitude of the generated potential [6].

Influence of the donor concentration on piezoelectric potential reduction and the external surface charges, have been investigated, based on electrostatic effect. The researchers had aimed to overcome the screening effect of the vertically compressed $\mathrm{ZnO}$ nanowire [6, 7]. However, the effects of mentioned parameters on conduction band changes, carrier concentration, and the magnitude and distribution of the piezoelectric potential have not been well understood. Thus, in this paper, by considering the wide range of donor concentrations and surface charges density, the influence of surface charges density on electrical energy, mechanical energy, and the nanogenerator efficiency are investigated using finite element method (FEM). Also the interplay of the donor concentration and surface charges at the top cross section of the nanowire are studied.

\section{MODEL DEFINITION}

Mechanical and electrical behavior of the $\mathrm{ZnO}$ piezoelectric nanogenerator is coupled via the following equation [8], which are used to calculate the stress $T$ and displacement $D$, using FEM:

$$
\begin{aligned}
& T=C_{E} S-e^{T} E, \\
& D=e S+\kappa E,
\end{aligned}
$$

\footnotetext{
*Tahere.fanaei@ece.usb.ac.ir
} 
where $S$ is strain tensor, $E$ is the electric field vector, $C_{E}$ is the elastic stiffness tensor, $e$ is the piezoelectric constant tensor, $\kappa$ is permittivity tensor, and $e^{T}$ is the transpose of the tensor $e$. The anisotropic elastic modulus tensor has been taken from the C6V symmetry of the $\mathrm{ZnO}$ crystal with wurtzite structure [8]. The numerical values of relative dielectric constants, piezoelectric constants and corresponding tensors are summarized in Table 1 [9]. In the following, two electrostatic equations are used. Considering the moderate conductivity in $\mathrm{ZnO}$ nanowire, the Gauss's law links the displacement $\mathrm{D}$ to the charge density, and the mechanical equation which links the stress $T$ to the applied force $F[9]$.

$$
\begin{gathered}
\nabla D=\rho_{V}=e\left(p-n+N_{D}^{+}-N_{A}^{-}\right) \\
-\nabla T=F
\end{gathered}
$$

In equation (2), $\mathrm{P}$ and $\mathrm{n}$ are related to the hole concentration in the valance band and the electron concentration in the conduction band, respectively. Also, $N_{D}^{+}$ is the ionized donor concentration, $N_{A}^{-}$is the ionized acceptor concentration, and $e$ is electron charge. It should be mentioned that for $\mathrm{ZnO} \mathrm{NW}$, with $n$-type doping concentration, $P=N_{A}^{-}=0$ [9].

Table 1 - Parameters for $\mathrm{ZnO}$ nanowire with wurtzite structure in $\mathrm{C}_{6 \mathrm{v}}$ symmetry [9]

\begin{tabular}{|l|l|l|l|}
\hline Parameter & $\begin{array}{l}\text { Magnitude } \\
{[\mathrm{GPa}]}\end{array}$ & Parameter & Magnitude \\
\hline C11 & 207 & e15 & $-0.45\left[\mathrm{C} / \mathrm{m}^{2}\right]$ \\
\hline C12 & 117.7 & e31 & $-0.51\left[\mathrm{C} / \mathrm{m}^{2}\right]$ \\
\hline C13 & 106.1 & e33 & $1.22\left[\mathrm{C} / \mathrm{m}^{2}\right]$ \\
\hline C33 & 209.5 & k 11 & 7.77 \\
\hline C44 & 44.8 & к 22 & 7.77 \\
\hline C55 & 44.6 & к 33 & 8.91 \\
\hline
\end{tabular}

The simulation is limited to the static analysis of the open circuit voltage. The nanowire is geometrically approximated as a cylinder with radius and length of $R=25 \mathrm{~nm}, L=600 \mathrm{~nm}$, along the $z$-coordinate. In the considered nanowire, the one end is affixed to a gold substrate, while the other end is free and is normally compressed by a vertical force $F=80 \mathrm{nN}$, exerted on the top cross section. The geometry is surrounded by free space (air) to provide the zero electric filed boundary condition at distance far away from the nanowire. Fig. 1, shows a 3D schematic of the geometry under analysis by considering some associated additional information. The $\mathrm{ZnO}$ nanowire is assumed with moderate conductivity and is doped with initial donor concentration $N D=1 \times 10^{17} \mathrm{C} / \mathrm{m}^{3}$. In $\mathrm{ZnO}$ nanowire under the external uniaxial compression, charges are polarized along the nanowire creating a dipole-like piezoelectric potential along the $c$-axis. Due to the positive piezoelectric coefficient of $\mathrm{ZnO}$ nanowire along the $c$-axis (d33), the top part of nanowire exhibits a negative potential relative to the ground potential at the bottom end [10].

\section{RESULTS AND DISCUSSION}

The potential distribution along the c-axis provides the electrical boundary conditions in two cases, which plays a vital role to improve the nanogenerator output. First, the bottom cross section of the nanowire is considered as ground and set to zero potential. In the second case, the bottom electrode with the initial thickness of $10 \mathrm{~nm}$ is connected to the ground. According to the results, which are shown in Fig. 2a, connecting the electrode to the ground leads to an additional voltage drop at the bottom end of the nanowire and consequently increases the voltage difference, compared to the nanowire bottom cross section grounded. While the voltage drop at the top end $(600 \mathrm{~nm})$ of $\mathrm{NW}$ has the same magnitude in both states. Similar behavior is also seen by [7].

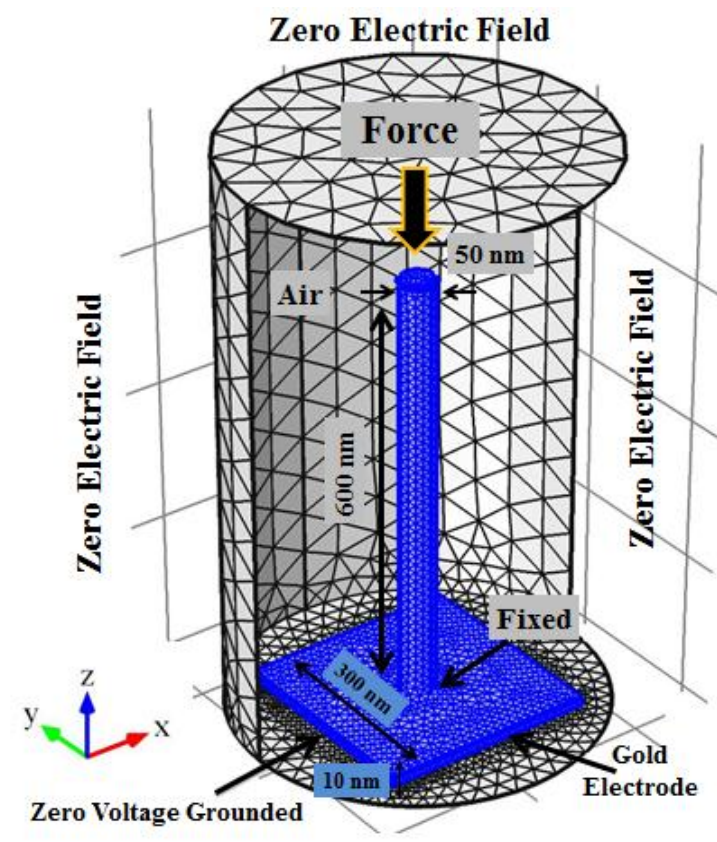

Fig. 1 - Schematic of the considered $\mathrm{ZnO}$ nanowire

Taking into account the grounded electrode as the electrical boundary condition, the influence of electrode thickness on the potential drop is investigated. The substrate thickness is altered from $5 \mathrm{~nm}$ to $150 \mathrm{~nm}$. From Fig. 2b, it is observed that the potential drop increases for thicknesses of $5 \mathrm{~nm}$ to $40 \mathrm{~nm}$ and then it becomes less effective for thickness larger than $40 \mathrm{~nm}$. Although an excessive rise of the electrode thickness does not have a significant impact on the potential, but from the application point of view the flexibility of the platform is reduced and it is undesirable for nanogenerators operation in some applications. In the next step, the donor concentrations ranging from $N D=1 \times 10^{15} \mathrm{C} / \mathrm{m}^{3}$ to $N D=1 \times 10^{18} \mathrm{C} / \mathrm{m}^{3}$ are added to the model. Fig. $3 \mathrm{a}$ and Fig. $3 \mathrm{~b}$, shows the conduction band variations and the distribution of the electron concentrations in the model. Fig. 3c, shows the generated open circuit voltages for the $\mathrm{ZnO}$ nanowire as functions of their donor concentrations. The force causes to emit the electrons away from the tip; the charge carriers are largely depleted from the top part of the $\mathrm{ZnO}$ nanowire and are accumulated at the bottom end, 

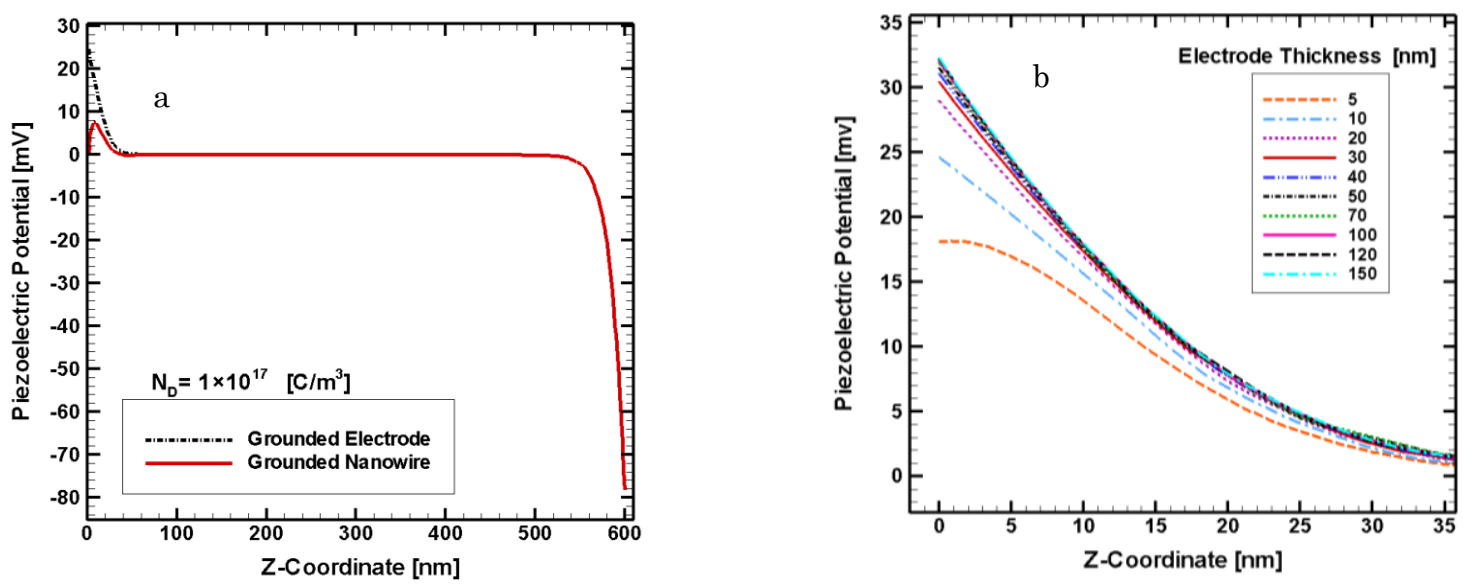

Fig. 2 - a) Piezoelectric potential along $\mathrm{ZnO}$ nanowire (NW) with grounded NW base and bottom electrode. b) Effect of the bottom electrode thickness on potential distribution at the bottom of $\mathrm{ZnO} \mathrm{NW}$
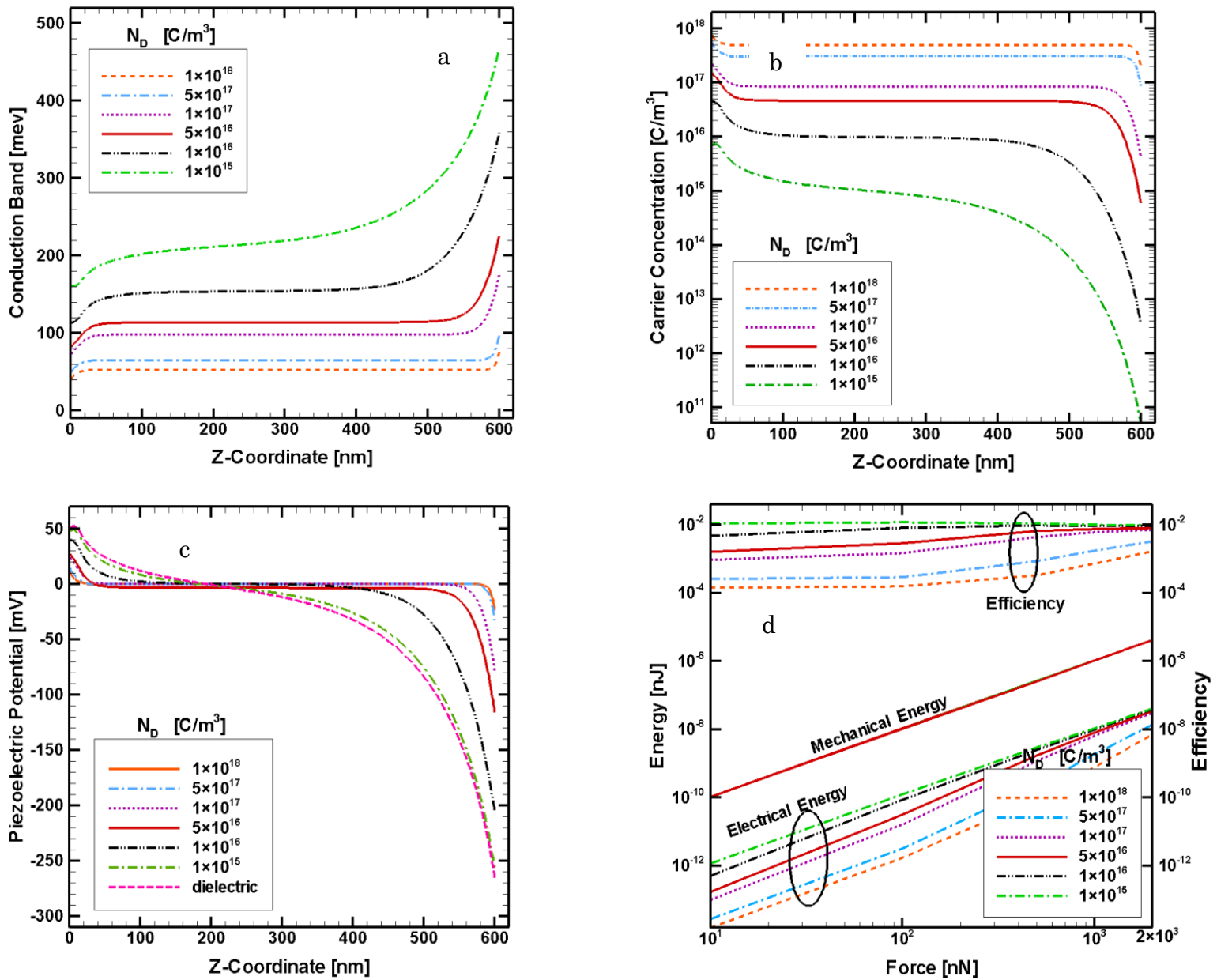

Fig. 3 - a) Conduction band and b) carrier concentration along the NW, in presence of the different donor concentration levels from $N D=1 \times 10^{15} \mathrm{C} / \mathrm{m}^{3}$ to $N D=1 \times 10^{18} \mathrm{C} / \mathrm{m}^{3}$. c) Piezoelectrice potential and d) electrical and mechanical energy and efficiency, as a function of force, for different donor concentrations from $N D=1 \times 10^{15} \mathrm{C} / \mathrm{m}^{3}$ to $N D=1 \times 10^{18} \mathrm{C} / \mathrm{m}^{3}$

so creates a depletion zone close to the tip. The conduction band at the top end of the nanowire goes up relative to the bottom end. The depletion region leads to the potential drop which is considered as the output piezoelectric potential.

The donor concentration, with its positive charge, leads to screen the negative charges and balance the potential along the nanowire. Therefore by increasing the donor concentration, the difference of the conduction band edges at the top and bottom ends of the nanowire is reduced and the conduction band tends to reach the zero level (Fig. 3a). Therefore, the results are well indicated that the free charge carriers and consequently the screening effect have significant impact on the profile of carrier concentration, conduction band variations and finally, on piezoelectric potential along 
the nanowire, and should carefully considered in each further experience. Increasing the impurity inside the NW causes to screen more amount of the negative piezoelectric potential and decreases the potential barrier height (Fig. 3c). The potential is almost completely screen for the donor concentration $N D=1 \times 10^{18} \mathrm{C} / \mathrm{m}^{3}$, and is approximately equal to $V=-25 \mathrm{mv}$. The procedure of decreasing the potential relative to increase the impurity is shown in Fig. 4. Electromechanical coupling efficiency parameter is of vital importance to NGs, which indicates the amount of mechanical energy that converts to electrical energy, proportional to the applied force. According to Fig. 3d, mechanical energy increases with increasing the force, independent of the donor concentration levels. However, the electrical energy varies with the donor concentrations and as ex-
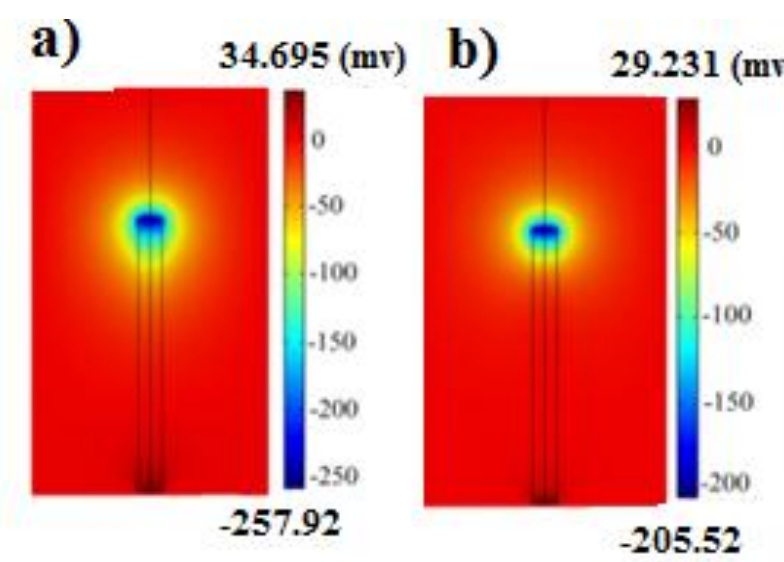

pected, the energy increases in lower donor concentrations (the potential is more screened in high donor levels). As a matter of fact, based on conventional electrical energy equation $\left(E=1 / 2 q^{2} / \mathrm{C}\right)$, the electrical energy is also proportional to the squared charge and more force makes the further charge polarization and finally causes the further output energy. The present simulations demonstrate that, the efficiency is more sensitive to the force in higher donor levels and becomes negligible at sufficiently low donor concentrations. Also as seen, the efficiency is higher at low donor levels (Fig. 3d). Results in the previous section showed that the existence of free charge carriers in $\mathrm{ZnO}$ nanowire has an undesirable impact in the piezoelectric NGs performance, and leads to decrease the potential so far.

Fig. 4 - Piezoelectric potential distribution along $\mathrm{ZnO} N W$ for: a) $N D=1 \times 10^{15} \mathrm{C}^{\mathrm{m}} \mathrm{m}^{3}$, b) $N D=1 \times 10^{16} \mathrm{C} / \mathrm{m}^{3}$, c) $N D=1 \times 10^{17} \mathrm{C} / \mathrm{m}^{3}$, and d) $N D=1 \times 10^{18} \mathrm{C} / \mathrm{m}^{3}$

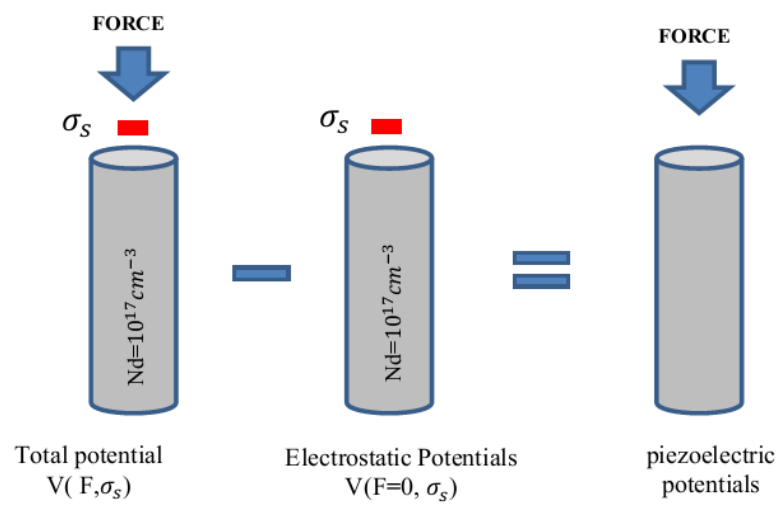

Fig. 5 - Calculation procedure for piezoelectric potential in presence of the external free charges on the top of the ZnO NW

In this part of paper in order to minimize the influence of screening effects and to compensate the reduction of piezoelectric potential, the electrostatic effect is used. By imposing the external surface charges on the top cross section of the $\mathrm{ZnO}$ nanowire, the repulsive force between charges inside the nanowire and the surface charges at the top of the nanowire, leads to deplete the charges in the upper part of the nanowire. The depletion region width significantly increases and consequently the negative piezoelectric potential along the nanowire increases. It is noteworthy that, the ultimate piezoelectric potential along the $\mathrm{z}$-axis is obtained by subtracting the total potential (the total potential is due to presence of external force and external surface

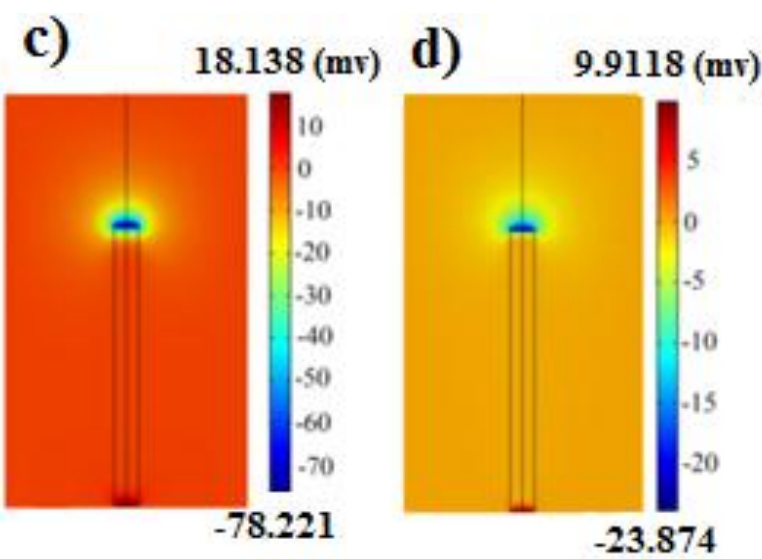



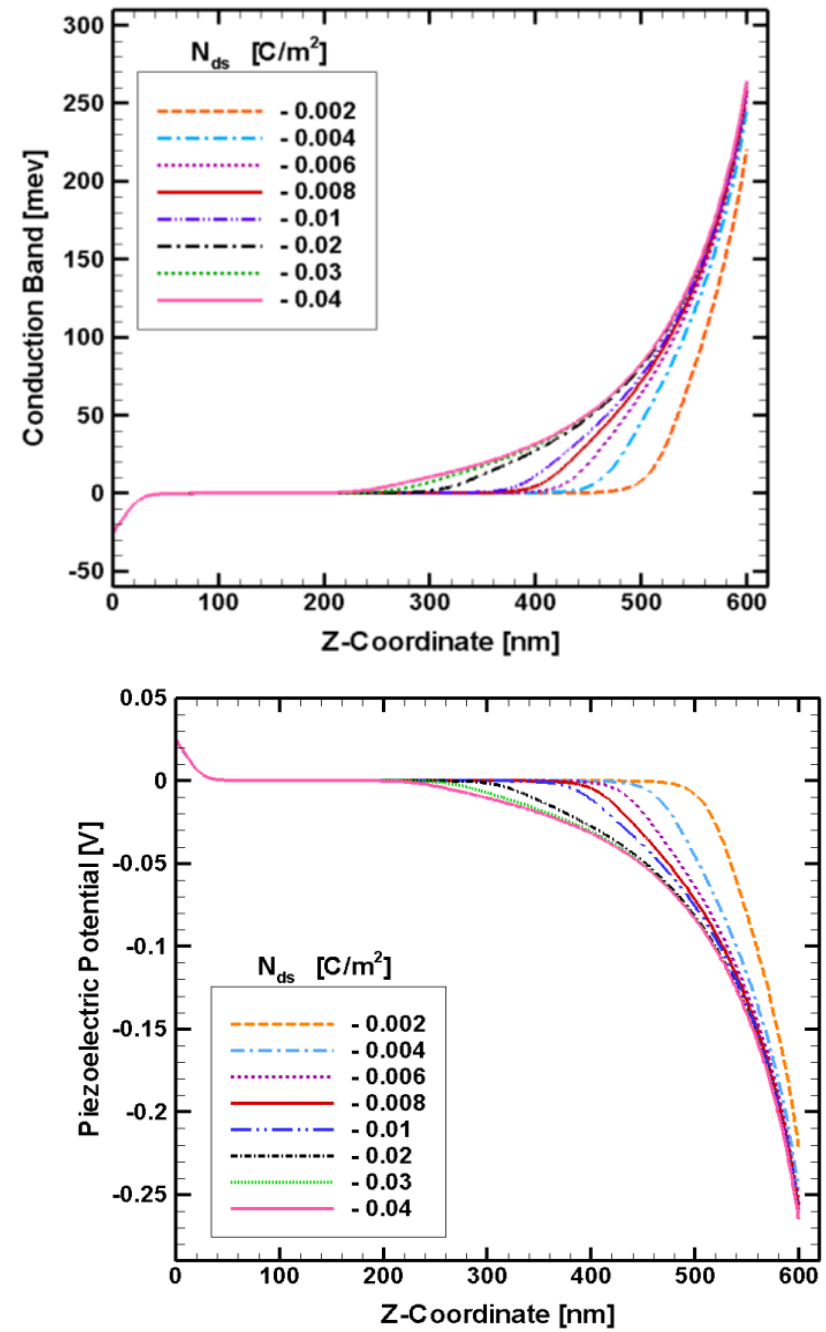
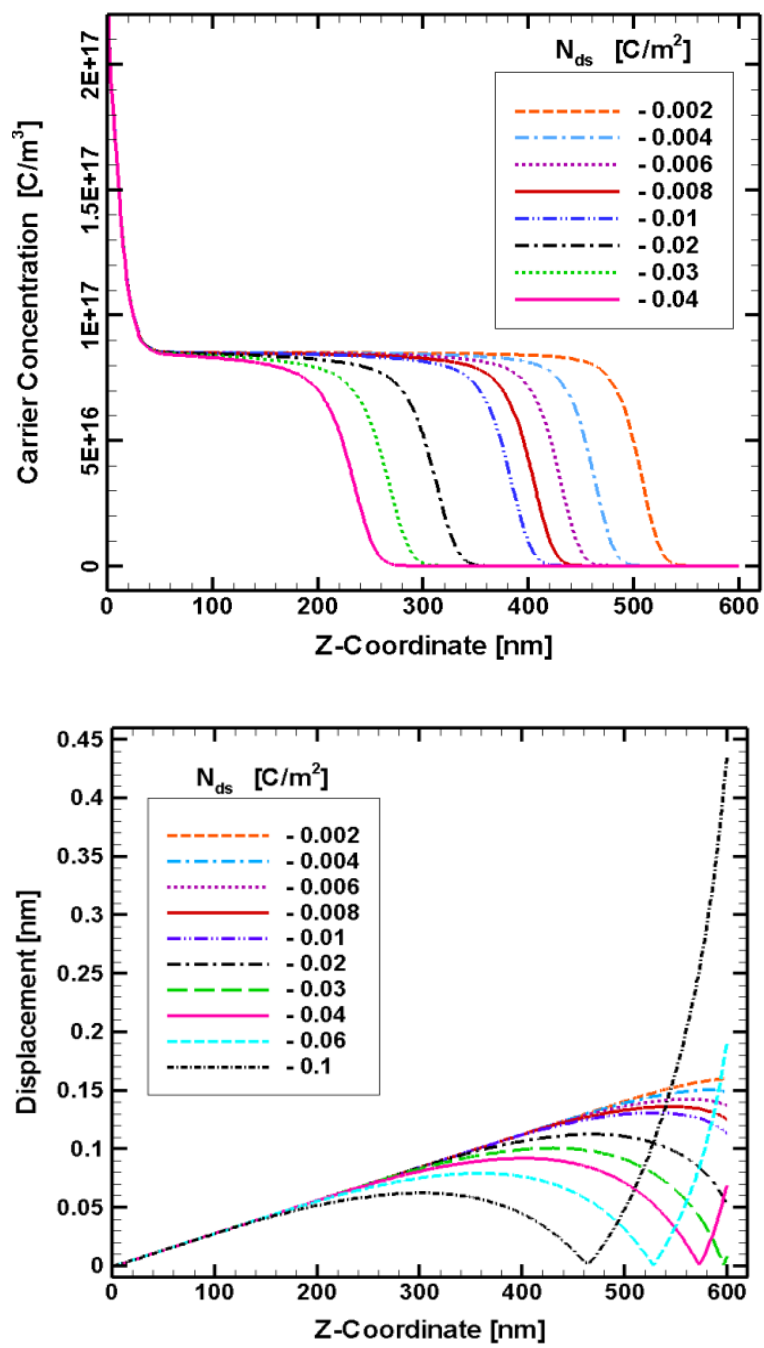

Fig. 6 - a) Conduction band and b) carrier concentration, along the NW, in presence of the different external surface free charges and the donor concentration of $N D=1 \times 10^{17} \mathrm{C} / \mathrm{m}^{3}$. c) Piezoelectric potential and d) displacement, along NW, in presence of the different external free charges and the donor concentration of $N D=1 \times 10^{17} \mathrm{C} / \mathrm{m}^{3}$

will reach to $400 \mathrm{~nm}$ for $N_{d s}=-0.04 \mathrm{C} / \mathrm{m}^{2}$. The minimum surface charge density causes to the $60 \mathrm{~nm}$ enhancement of the depletion zone width relative to the similar case $\left(N D=1 \times 10^{17} \mathrm{C} / \mathrm{m}^{3}\right)$ without considering the electrostatic effect.

According to the Fig. 6a, the external charges on the top surface of the nanowire have caused the conduction band reaches the zero level (Fermi level) and provide a relatively high potential barrier compare to the nanowire without surface charge. The potential barrier height is $220 \mathrm{mEv}$ for the nanowire with $N_{d s}=-$ $0.002 \mathrm{C} / \mathrm{m}^{2}$ and $N D=1 \times 10^{17} \mathrm{C} / \mathrm{m}^{3}$ and it is $80 \mathrm{mEv}$ for $N D=1 \times 10^{17} \mathrm{C} / \mathrm{m}^{3}$ without surface charges (Fig. 3a).

Such an increasing on the potential barrier height augments the output potential. The displacement diagram along the NW is shown in Fig. 6d. Nanowire has a reverse displacement procedure for charges above the $N_{d s}=-0.03 \mathrm{C} / \mathrm{m}^{2}$, and the piezoelectric potential is approximately saturated in this level. According to the profile patterns of the potential (Fig. 6c), the piezoelectric potential is constant for $N_{d s}>-0.03 \mathrm{C} / \mathrm{m}^{2}$.

Fig. 7a, shows the magnitude of piezoelectric potential at the tip of nanowire versus the surface charge densities $\left(-0.002 \mathrm{C} / \mathrm{m}^{2}<N_{d s}<-0.08 \mathrm{C} / \mathrm{m}^{2}\right)$ for the different donor concentrations $\left(1 \times 10^{15} \mathrm{C} / \mathrm{m}^{3}<\mathrm{ND}<1 \times 10^{18} \mathrm{C} / \mathrm{m}^{3}\right)$. Piezoelectric potential is differently saturated for the various donor concentration levels. The electrostatic effect has much more influence on improving the potential within $\mathrm{ZnO}$ NWs with high donor levels. Whereas the electrostatic effect does not have a significant effect in nanowire with $N D=1 \times 10^{15} \mathrm{C} / \mathrm{m}^{3}$. In comparison with energy and efficiency diagram in previous section (Fig. $3 \mathrm{~d}$ ), the results obtained from electrostatic effect are different (Fig. 7b). At the low exerted forces the mechanical energy depends to the electrostatic charges while it is obviously not affected by surface charges at the forces above $500 \mathrm{nN}$. The dependency of electrical energy to the surface charges is more important than the influence of the forces on increasing the depletion region at the top of the nanowire. Thus, the electrical energy for the minimum force $(F=10 \mathrm{nN})$ and for the maximum force $(F=2 \mu \mathrm{N})$, is not significantly changed. However, increasing the surface charge density leads to rise up the electrical energy levels. Since the electrical energy levels are improved due to the surface charges, the efficiency slightly decreases when the mechanical energy is higher than the electrical energy levels. In overall, the electrical energy and efficiency are increased by applying the electrostatic effect. 

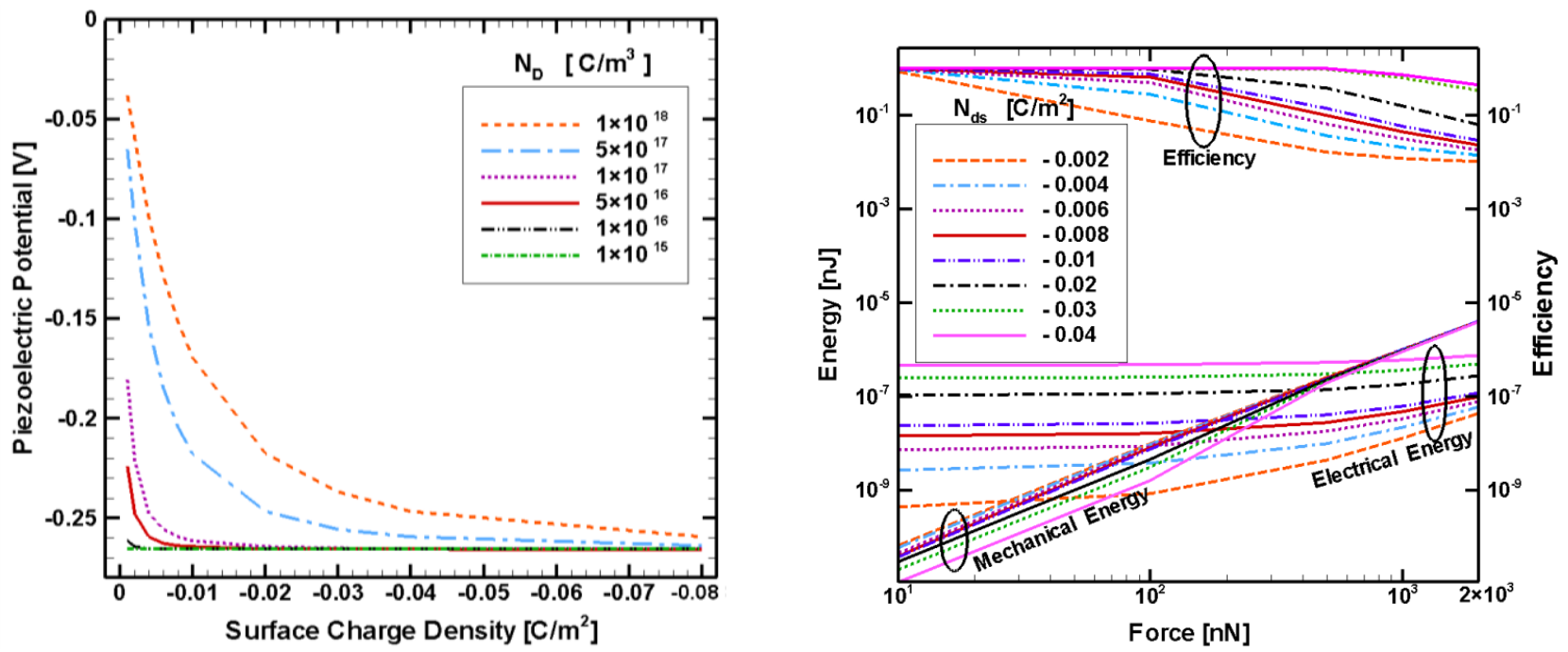

Fig. 7 - a) Maximum piezoelectric potential at the tip of $\mathrm{ZnO}$ NW as a function of surface charge density, for different donor concentration, and b) Electrical and mechanical energy and efficiency as a function of force, for different surface charge density

\section{CONCLUSION}

In this work, the interaction between donor concentration and surface charges at the tip of $\mathrm{ZnO} \mathrm{NW}$ is investigated. The electrostatic effect reduces the undesirable effect of free charges in $\mathrm{ZnO} \mathrm{NW}$. The results obtained from simulation are a guideline for realizing high efficient nanogenerators. The external charges more than $N_{d s}=-0.03 \mathrm{C} / \mathrm{m}^{2}$, for nanowire with $N D=1 \times 10^{17} \mathrm{C} / \mathrm{m}^{3}$, saturate the piezoelectric potential and change the nanowire compression procedure. Also, it is seen that the influence of the external negative surface charges on $\mathrm{ZnO} \mathrm{NW}$ with higher donor concentration is more important. Existence of the surface charges affect the electrical and mechanical behavior of the device, and therefore, improve the nanogenerator efficiency. The present results are helpful for improving

\section{REFERENCES}

1. Z.L. Wang, Nanogenerators for Self-powered Devices and Systems (Georgia Institute of Technology: Atlanta, USA: 2011).

2. Z.L. Wang, J. Song, Science 312, 242 (2006).

3. Zh. Wang, X. Pan, Y. He, Y. Hu, H. Gu, Y. Wang, Adv. Mater. Res. 2015, 1 (2015).

4. R Hinchet, S. Lee, G. Ardila, L. Montès, M. Mouis, Zh.L. Wang, Adv. Funct. Mater. 24, 971 (2014).

5. R. Hinchet, S. Lee, G. Ardila, L. Montès, M. Mouis, L.Zh. Wang, J. Energ. Power Eng. 7, 1816 (2013). the performance of NGs via controlling of the surface and volume charges of the $n$-type $\mathrm{ZnO} \mathrm{NW}$.

\author{
ABBREVIATIONS \\ $\mathrm{AFM}=$ Atomic force microscopy \\ FEM = Finite element method \\ $\mathrm{NG}=$ Nanogenerator \\ $\mathrm{NW}=$ Nanowire \\ $\mathrm{ZnO}=$ Zinc oxide
}

\section{ACKNOWLEDGMENT}

The authors thank the Ministry of Science, Research and Technology of I.R. Iran for its financial support.

6. S.M. Kim, H. Kim, Y. Nam, S. Kim, AIP Adv. 2, 042174 (2012).

7. G. Romano, G. Mantini, A.D. Carlo, A. D'Amico, Ch. Falconi, Z.L. Wang, Nanotechnology 22, 465401 (2011).

8. Y. Gao, Z.L. Wang, Nano Lett. 7, 2499 (2007).

9. Y. Gao, Z.L. Wang, Nano Lett. 9, 1103 (2009).

10. S.M. Kim, J.L. Sohn, H.J. Kim, J. Ku, Y.J. Park, S.N. Cha, J.M. Kim, Appl. Phys. Lett. 101, 013104 (2012). 